\title{
Maf1-mediated repression of RNA polymerase III transcription inhibits tRNA degradation via RTD pathway
}

\author{
TOMASZ W. TUROWSKI, ${ }^{1}$ IWONA KARKUSIEWICZ, ${ }^{2}$ JUSTYNA KOWAL, ${ }^{2}$ and MAGDALENA BOGUTA ${ }^{1,2,3}$ \\ ${ }^{1}$ Institute of Biotechnology, Faculty of Chemistry, Warsaw University of Technology, 00-664 Warsaw, Poland \\ ${ }^{2}$ Institute of Biochemistry and Biophysics, Polish Academy of Sciences, 02-106 Warsaw, Poland
}

\begin{abstract}
tRNA precursors, which are transcribed by RNA polymerase III, undergo end-maturation, splicing, and base modifications. Hypomodified tRNAs, such as tRNA $^{\text {Val(AAC) }}$, lacking 7-methylguanosine and 5-methylcytidine modifications, are subject to degradation by a rapid tRNA decay pathway. Here we searched for genes which, when overexpressed, restored stability of

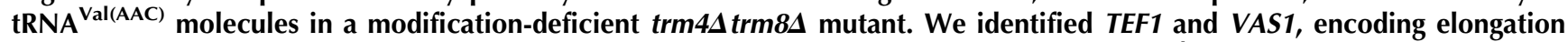
factor eEF1A and valyl-tRNA synthetase respectively, which likely protect hypomodified tRNA ${ }^{\text {Val(AAC) }}$ by direct interactions. We also identified MAF1 whose product is a general negative regulator of RNA polymerase III. Expression of a Maf1-7A mutant that constitutively repressed RNA polymerase III transcription resulted in increased stability of hypomodified tRNA ${ }^{\text {Val(AAC) }}$. Strikingly, inhibition of tRNA transcription in a Maf1-independent manner, either by point mutation in RNA polymerase III subunit Rpc128 or decreased expression of Rpc17 subunit, also suppressed the turnover of the hypomodified tRNA ${ }^{\text {Val(AAC) }}$. These results support a model where inhibition of tRNA transcription leads to stabilization of hypomodified tRNA ${ }^{\text {Val(AAC) }}$ due to more efficient protection by tRNA-interacting proteins.
\end{abstract}

Keywords: rapid tRNA decay; tRNA; RNA polymerase III; tRNA transcription; Maf1; translation elongation factor

\section{INTRODUCTION}

Regulation of the tRNA transcription machinery and the post-transcriptional steps that generate functional tRNAs are of fundamental importance for protein biosynthesis and cell survival. tRNAs precursors are transcribed by RNA polymerase III (Pol III), a large enzyme consisting of 17 subunits and requiring several auxiliary factors. tRNA transcription needs to be controlled in response to nutrient availability and other environmental circumstances; up-regulation of tRNA transcription can promote cell proliferation and transformation as well as tumorigenesis in mice (Marshall et al. 2008). Mammalian Pol III is controlled by the tumor suppressors p53 and $\mathrm{Rb}$, and Maf1 protein, which is conserved also in all eukaryotes (Pluta et al. 2001; White 2004). In yeast, Maf1 is the only known general negative Pol III regulator which ensures coupling of tRNA biosynthesis to cell growth and metabolism (Boguta and Graczyk 2011).

\footnotetext{
${ }^{3}$ Corresponding author

E-mail magda@ibb.waw.pl

Article published online ahead of print. Article and publication date are at http://www.rnajournal.org/cgi/doi/10.1261/rna.033597.112.
}

Primary tRNA transcripts are extended at the $5^{\prime}$ and $3^{\prime}$ termini and may contain introns. Processing of the $5^{\prime}$ leader and trimming of the $3^{\prime}$ trailer occur in the nucleus and are followed by CCA addition at the newly formed $3^{\prime}$ termini. In yeast, the end-processed tRNAs are transported to the cytoplasm where introns are removed (for review, see Phizicky and Hopper 2010). The nuclear export of yeast tRNA is regulated by environmental conditions in coordination with Maf1-mediated transcription control, thereby coupling tRNA synthesis and maturation (Karkusiewicz et al. 2011).

In addition to the processing at the termini and intron removal, tRNAs undergo nucleotides modification, both in the nucleus and in the cytoplasm. Modifications in or around the anticodon loop affect translation and cell growth, while some body tRNA modifications contribute to its folding and stability (Chernyakov et al. 2008a). Inappropriately modified or folded tRNAs can be the target of at least two turnover mechanisms. Pre-tRNA $\mathrm{i}^{\mathrm{Met}}$ lacking $\mathrm{m}^{1} \mathrm{~A}$ is destroyed by the nuclear surveillance pathway in which the pre-tRNA is first polyadenylated by the TRAMP complex, and then degraded from the 3 ' end by the nuclear exosome (Anderson and Parker 1998; Kadaba et al. 2004; Kuai et al. 2004; Wyers et al. 2005). Another pathway, called rapid tRNA decay (RTD), causes partial loss of the hypomodified tRNA in mutant 
lacking specific modifications (Alexandrov et al. 2006; Kotelawala et al. 2008). One substrate for the RTD pathway is tRNA ${ }^{\text {Val(AAC) }}$, lacking the $\mathrm{m}^{7} \mathrm{G}_{46}$ and $\mathrm{m}^{5} \mathrm{C}_{49}$ modifications. This tRNA is degraded at an elevated temperature by $5^{\prime} \rightarrow 3^{\prime}$ exonucleases, Xrn1 and Rat 1 (Chernyakov et al. 2008b). A more recent study has demonstrated that lack of specific modifications leads to instability of the tRNA acceptor stem and exposes the 5' end of the tRNA molecule to degradation by Xrn1 nuclease (Whipple et al. 2011). Instability of acceptor stem causes tRNA degradation mediated by CCACCA addition at the $3^{\prime}$ end (Wilusz et al. 2011).

Here we show that degradation of hypomodified tRNA is prevented when tRNA transcription is decreased. Inhibition of Pol III activity in trm $4 \Delta$ trm $8 \Delta$ cells lacking the $\mathrm{m}^{7} \mathrm{G}_{46}$ and $\mathrm{m}^{5} \mathrm{C}_{49}$ modifications in their tRNAs resulted in lower degradation of $\mathrm{tRNA}{ }^{\mathrm{Val}(\mathrm{AAC})}$. A similar effect, i.e., stabilization of hypomodified tRNA ${ }^{\mathrm{Val}(\mathrm{AAC})}$, could be achieved in the trm $4 \Delta$ trm $8 \Delta$ mutant by overexpression of genes encoding translation elongation factor eEF1A or valyl-tRNA synthetase, two tRNA-interacting proteins. According to our model, in the context of a globally lower tRNA level, tRNA $^{\text {Val(AAC) }}$ competes more efficiently for tRNA-interacting proteins which help it achieve proper conformation and/or protect from nucleases.

\section{RESULTS}

\section{Identification of library plasmids that overcome growth defect related to degradation of $t R N A^{\text {Val(AAC) }}$}

Our rationale was that degradation of hypomodified tRNA $^{\mathrm{Val}(\mathrm{AAC})}$ could be limited in vivo by some factors or as yet undescribed activities affecting the RTD pathway. In an attempt to identify such putative factors increasing stability of hypomodified tRNA, we used a genetic strategy employing the growth phenotype of the trm $4 \Delta$ trm $8 \Delta \mathrm{mu}$ tant, which is not viable at $37^{\circ} \mathrm{C}$ due to degradation of the tRNA ${ }^{\mathrm{Val}(\mathrm{AAC})}$ lacking the $\mathrm{m}^{7} \mathrm{G}_{46}$ and $\mathrm{m}^{5} \mathrm{C}_{49}$ modifications (Chernyakov et al. 2008b). We therefore looked for multicopy suppressors of the trm $4 \Delta$ trm $8 \Delta$ growth deficiency at an elevated temperature.

The trm4 trm8s strain was transformed with a yeast genomic library based on the pFL44L multicopy vector (Stettler et al. 1993). Sixty-five transformants growing at an elevated temperature $\left(37^{\circ} \mathrm{C}\right)$ were selected from $\sim 5 \times 10^{3}$ transformed cells. For each of them we determined whether their temperature resistance required the presence of the library plasmid. Twenty-nine candidates showed the dependence of the suppression level upon the presence of the plasmid. Sequencing of the plasmids from those cells identified 16 inserts containing various $\mathrm{RRA}^{\mathrm{Val}(\mathrm{AAC})}$ genes and 13 other inserts. Selection of genomic inserts containing tRNA $^{\mathrm{Val}(\mathrm{AAC})}$ genes was expected in our screen since complementation of the thermosensitive ( $t s)$ phenotype of trm $4 \Delta$ trm $8 \Delta$ mutant by an overdose of the tRNA ${ }^{\operatorname{Val}(A A C)}$ gene had been reported previously (Alexandrov et al. 2006). All the other plasmids were retransformed into trm $4 \Delta$ trm $8 \Delta$ and assayed for growth at an elevated temperature. Sequencing and subsequent subcloning of the suppressor plasmids led to the identification of five genes: TEF1, VAS1, $M A F 1, R V B 2$, and POP5. One plasmid contained a $5^{\prime}-$ terminal part of the RPC160 gene encoding the largest subunit of RNA polymerase III.

TEF1 encodes the translation elongation factor eEF1A. This protein is expressed from two genes in the yeast genome, TEF1 and TEF2, which contain identical ORFs. eEF1A is the eukaryotic homolog of Escherichia coli EF-Tu and binds in a GTP-dependent manner to aminoacyl tRNAs to deliver them to the ribosome (Mateyak and Kinzy 2010). We found that not only TEF1 but also TEF2 suppressed the

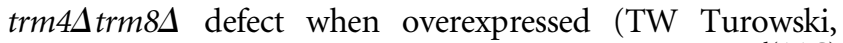
unpubl.). Thus, the phenotypic effect of the tRNA ${ }^{\text {Val(AAC) }}$ instability could be compensated by an overdosage of eEF1A for augmenting the eEF1A-tRNA interaction.

Interestingly, eEF1A was not the only tRNA-binding protein among the identified suppressors (Fig. 1). VAS1 encodes valyl-tRNA synthetase, which interacts with tRNA ${ }^{\text {Val }}$ directly. This suggested that the temperature resistance of trm $4 \Delta$ trm $8 \Delta$ brought about by high level of VAS1 expression could likewise be due to stabilization of the hypomodified $\mathrm{tRNA}^{\mathrm{Val}(\mathrm{AAC})}$ by the interacting protein. Such an interaction could help the $\mathrm{RRNA}^{\mathrm{Val}(\mathrm{AAC})}$ achieve a correct conformation despite the lack of proper modifications or could simply mechanistically protect it from the degradation machinery.

To address specificity of RTD suppression by tRNAinteracting proteins, we introduced TEF1 and VAS1 plasmids to tan $1 \Delta$ trm $44 \Delta$ mutant. The lack of Tan 1 and Trm44 results in growth defect at $37^{\circ} \mathrm{C}$ due to degradation of $\mathrm{tRNA}^{\mathrm{Ser}(\mathrm{CGA})}$ and $\mathrm{tRNA}^{\mathrm{Ser}(\mathrm{UGA})}$ missing $\mathrm{Um}_{44}$ and $\mathrm{ac}^{4} \mathrm{C}_{12}$ modifications (Kotelawala et al. 2008). The temperaturesensitive phenotype of tan $1 \Delta \operatorname{trm} 44 \Delta$ was suppressed by TEF1 but not by VAS1 overdose (Supplemental Fig. S1). We thus confirmed that VAS1 suppresses RTD-related phenotype of trm $4 \Delta$ trm $8 \Delta$ by specifically affecting the tRNA $^{\text {Val(AAC) }}$ levels.

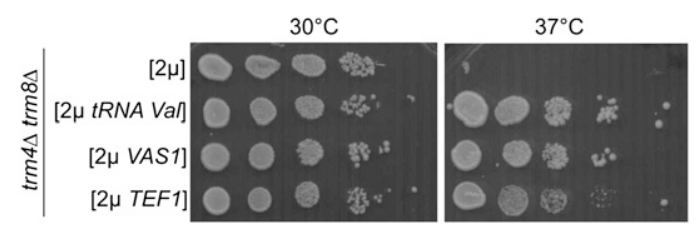

FIGURE 1. Multicopy suppressors of trm $4 \Delta$ trm $8 \Delta$ defect encoding known tRNA-interacting proteins. trm $4 \Delta$ trm $8 \Delta$ cells were transformed with multicopy plasmid pFL44L carrying VAS1 or TEF1. tRNA $^{\mathrm{Val}(\mathrm{AAC})}$ gene as a positive control and empty plasmid as a negative control. Transformants were grown overnight at $30^{\circ} \mathrm{C}$ in SD-ura liquid media, diluted to $\mathrm{OD}_{600}=1.0$ and serially 10 -fold diluted, spotted on YPD plates, and incubated at $30^{\circ} \mathrm{C}$ or $37^{\circ} \mathrm{C}$ for $2 \mathrm{~d}$. 
Next we examined the effects of TEF1 or VAS1 overdose on the stability of the hypomodified tRNA ${ }^{\text {Val(AAC) }}$ Using Northern blotting we monitored the degradation of tRNA $^{\mathrm{Val}(\mathrm{AAC})}$ following a shift of trm $4 \Delta$ trm $8 \Delta$ cells to an elevated temperature (Fig. 2). The level of Pol I-synthesized 5.8S rRNA served as an internal control. As published before (Chernyakov et al. 2008b), tRNA ${ }^{\text {Val(AAC) }}$ lacking the $\mathrm{m}^{7} \mathrm{G}_{46}$ and $\mathrm{m}^{5} \mathrm{C}_{49}$ modifications is rapidly degraded at $37^{\circ} \mathrm{C}$ (Fig. 2). Importantly, overexpression of TEF1 or VAS1 suppressed the tRNA ${ }^{\mathrm{Val}(\mathrm{AAC})}$ loss. Thus, an overdose of eEF1A or valyl-tRNA synthetase suppresses phenotype of trm $4 \Delta$ trm $8 \Delta$ mutant by preventing degradation of hypomodified tRNA ${ }^{\mathrm{Val}(\mathrm{AAC})}$ at an elevated temperature.

Whether the hypomodified tRNA ${ }^{\mathrm{Val}(\mathrm{AAC})}$ also interacts with proteins encoded by the other suppressors of the

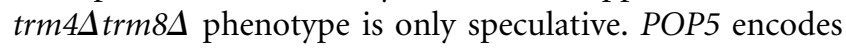
a subunit of RNAse $\mathrm{P}$ which cleaves tRNA precursors to generate mature 5' ends. Rvb2, a component of Rvb1/Rvb2 reptin complex, is essential in the assembly of several macromolecular complexes in transcription regulation and chromatin remodeling (Jha and Dutta 2009). The suppression by POP5 and RVB2 may be therefore indirect. Here, we have focused on two of the suppressors identified, TEF1 and VAS1, as their encoded proteins were known tRNA

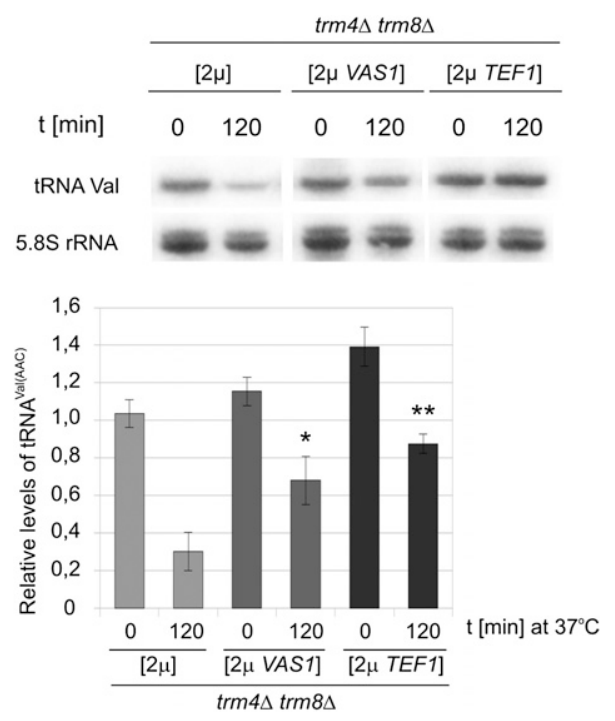

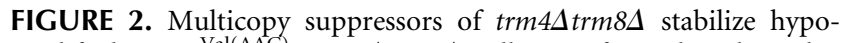
modified tRNA ${ }^{\mathrm{Val}(\mathrm{AAC})}$. trm $4 \Delta$ trm $8 \Delta$ cells transformed with multicopy plasmid carrying either VAS1 [ $2 \mu$ VAS1] or TEF1 [ $2 \mu$ TEF1] or empty plasmid $[2 \mu]$ were grown at $23^{\circ} \mathrm{C}$ and shifted to $37^{\circ} \mathrm{C}$ for $2 \mathrm{~h}$. RNA was isolated and analyzed by Northern hybridization with tRNA $^{\text {Val(AAC) }}$ probe and 5.8S rRNA probe (loading control). For quantification of tRNA $^{\mathrm{Val}(\mathrm{AAC})}$ levels were normalized to $5.8 \mathrm{~S}$ rRNA. Bars represent magnitude of $\mathrm{RNA}^{\mathrm{Val}(\mathrm{AAC})}$ change calculated relative to expression in trm $4 \Delta$ trm $8 \Delta$ cells bearing empty plasmid at 0 time point. Standard deviation (SD) was calculated on the basis of three independent experiments. $P$-values $-P<0.01$ for the probe indicated with a single asterisk $\left.{ }^{*}\right)$ and $P<0.005$ for the probe indicated with two asterisks $\left(^{* *}\right)$-were calculated relative to control strain transformed with empty plasmid $[2 \mu]$ at 120 time point. interactors. The remaining suppressors are of interest as well and will be studied later.

\section{Hypomodified tRNA $^{\text {Val(AAC) }}$ is stabilized by Maf1 overproduction}

Identification of the gene encoding Maf1 as a high copy suppressor of the trm $4 \Delta$ trm $8 \Delta$ phenotype was surprising (Fig. 3A). Mafl is a global negative regulator of Pol III transcription which, in an active state, decreases pre-tRNA synthesis (Pluta et al. 2001; Upadhya et al. 2002). Simple overexpression of MAF1 gene has little effect on the Pol III transcription (Desai et al. 2005) since activation of Maf1 requires its dephosphorylation (Oficjalska-Pham et al. 2006). By employing Northern blotting we confirmed that the steady state level of hypomodified tRNA ${ }^{\mathrm{Val}(\mathrm{AAC})}$ was actually not changed by increased expression of Mafl-encoding gene (Fig. 3B). The molecular mechanism of trm $4 \Delta$ trm $8 \Delta$ suppression by overexpression of $M A F 1$ is therefore not related to a compensatory synthesis of $\mathrm{tRNA}^{\mathrm{Val}(\mathrm{AAC})}$. In contrast, an overdose of the tRNA ${ }^{\mathrm{Val}(\mathrm{AAC})}$ gene more than doubled the steady state level of this tRNA at the permissive temperature (Fig. 3C).

To evaluate the effect of Mafl on the RTD pathway we monitored the degradation of $\mathrm{tRNA}^{\mathrm{Val}(\mathrm{AAC})}$ following a shift of trm $4 \Delta$ trm8 cells to an elevated temperature (Fig. 3B). Importantly, $M A F 1$ overexpression partially suppressed the tRNA $^{\text {Val(AAC) }}$ loss. Quantification of the Northern blots revealed that after $2 \mathrm{~h}$ at the elevated temperature tRNA ${ }^{\text {Val(AAC) }}$ is present at only $20 \%$ of the level before temperature shift in the trm $4 \Delta$ trm $8 \Delta$ strain transformed with a control plasmid. In contrast, in the trm $4 \Delta$ trm $8 \Delta$ strain transformed with a Mafl-encoding multicopy plasmid, the $\mathrm{RNA}^{\mathrm{Val}(\mathrm{AAC})}$ level was $40 \%$ of the level before the temperature shift (twofold more than without overexpression of Maf1). An overnight incubation at $37^{\circ} \mathrm{C}$ results in a complete loss of tRNA ${ }^{\mathrm{Val}(\mathrm{AAC})}$ and death of trm $4 \Delta$ trm $8 \Delta$ cells, both suppressed by MAF1 overdose (Fig. 3A; Supplemental Fig. S2).

In summary, overproduction of Maf1, a Pol III repressor, affords limited stabilization of hypomodified tRNA ${ }^{\text {Val(AAC) }}$.

\section{Maf1-7A mutant, a constitutively active Pol III repressor, stabilizes hypomodified tRNA ${ }^{\text {Val(AAC) }}$}

Identification of Mafl-encoding gene in the screen for proteins involved in stabilization of hypomodified tRNA ${ }^{\text {Val(AAC) }}$ raised the question of the mode of action of Maf1. Maf1 shuttles between the nucleus and the cytoplasm (Towpik et al. 2008). Its function in the cytoplasm is unknown, but one possibility is that cytoplasmic Maf1 could be directly involved in tRNA stabilization. However, our attempts to identify a direct Mafl-tRNA ${ }^{\text {Val(AAC) }}$ interaction failed (I Karkusiewicz and TW Turowski, unpubl.). We therefore explored the possibility of an indirect role of Maf1 in tRNA stabilization deriving from the known negative effect of Maf1 
A

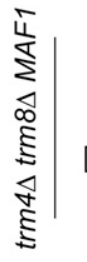

B

C

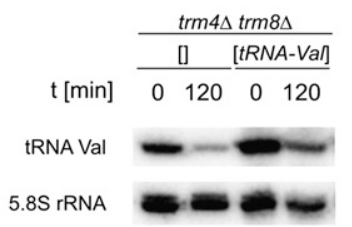

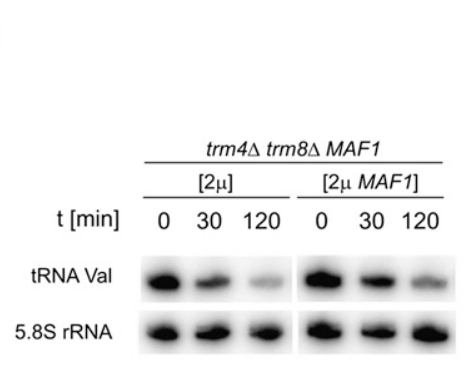

$30^{\circ} \mathrm{C}$

$[2 \mu]$
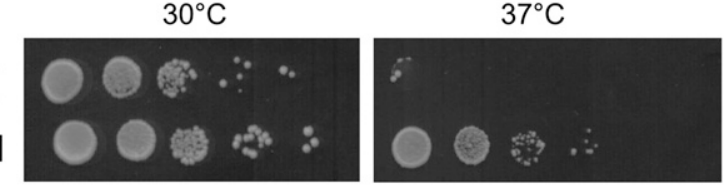

$[2 \mu M A F 1]$

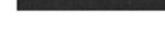

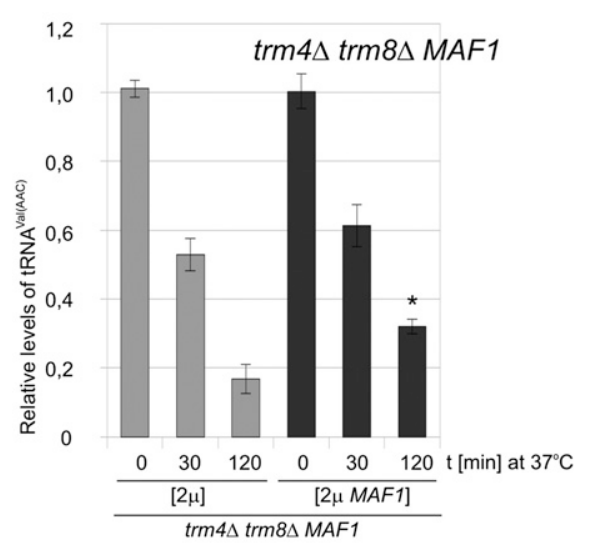

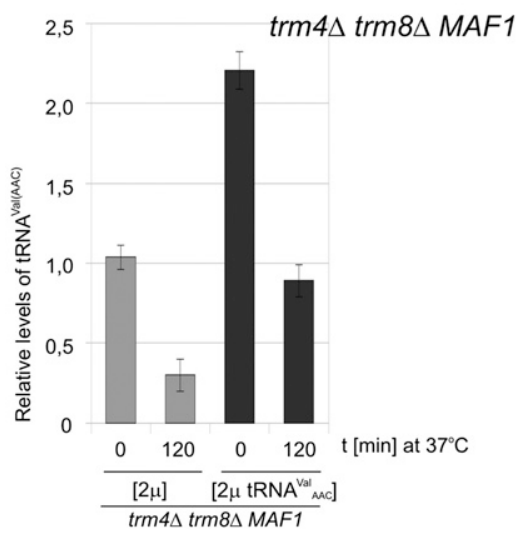

FIGURE 3. Overproduction of Maf1 suppresses thermosensitive phenotype and stabilizes hypomodified tRNA ${ }^{\mathrm{Val}(\mathrm{AAC})}$ in trm $4 \Delta$ trm $8 \Delta$ cells. (A) trm $4 \Delta$ trm $8 \Delta$ cells were transformed with multicopy pFL44L plasmid with MAF1 [2 $\mu$ MAF1] or empty plasmid $[2 \mu]$. Transformants were grown overnight at $30^{\circ} \mathrm{C}$ in SD-ura liquid media, diluted to $\mathrm{OD}_{600}=1.0$ and serially 10-fold diluted, spotted on YPD plates, and incubated at $30^{\circ} \mathrm{C}$ or $37^{\circ} \mathrm{C}$ for $2 \mathrm{~d}$. (B) trm $4 \Delta$ trm $8 \Delta$

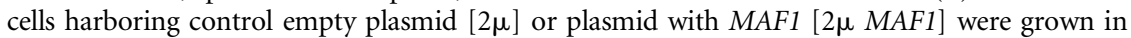
SC-ura medium at $30^{\circ} \mathrm{C}$ to $\mathrm{OD}=1.0$, shifted to $37^{\circ} \mathrm{C}$, and incubated for the indicated time. RNA was isolated and analyzed by Northern hybridization with tRNA ${ }^{\text {Val(AAC) }}$ probe and $5.8 \mathrm{~S}$ rRNA probe (loading control). For quantification of $\mathrm{tRNA} \mathrm{Al}^{\mathrm{Val}(\mathrm{AAC})}$ levels were normalized to $5.8 \mathrm{~S}$ rRNA. Bars represent magnitude of $t R N A^{\text {Val }(\mathrm{AAC})}$ change calculated relative to expression in trm $4 \Delta$ trm $8 \Delta$ cells bearing empty plasmid at 0 time point. SD was calculated on the basis of three independent experiments. $P$-value $<0.01$ for the indicated probe $\left.{ }^{*}\right)$ was calculated relative to control strain transformed with empty plasmid $[2 \mu]$ at 120 time point. (C) trm4trm $8 \Delta$ transformants with multicopy plasmid carrying gene coding tRNA ${ }^{\text {Val (AAC) }}\left[2 \mu t R N A^{\text {Val }(A A C)}\right]$ or empty plasmid $[2 \mu]$ were grown at $23^{\circ} \mathrm{C}$ and shifted to $37^{\circ} \mathrm{C}$ for $2 \mathrm{~h}$, RNA was isolated and analyzed by Northern hybridization as in $B$. Relative levels of tRNA ${ }^{\text {Val(AAC) }}$ were quantified (note that the scale is different than in $B$ ).

on tRNA transcription. We expected that an enhancement of the Maf1-mediated repression by its dephosphorylation would promote stabilization of hypomodified tRNA ${ }^{\text {Val(AAC) }}$. We therefore used the constitutively dephosphorylated Maf17A mutant (Huber et al. 2009). Cells expressing Maf1-7A had reduced basal tRNA levels due to an increased capacity of
Maf1-7A to bind and inhibit Pol III (Huber et al. 2009). First we verified the functionality of Maf1-7A encoded by a centromeric plasmid by genetic complementation (Supplemental Fig. S3A). Then the dephosporylated state of Maf17A was verified under our experimental conditions. Crude extracts were prepared from trm $4 \Delta$ trm $8 \Delta$ mafl $1 \Delta$ cells expressing Maf1-7A from a centromeric plasmid. Differentially phosphorylated forms of Maf1 were resolved by SDSPAGE and identified by immunoblotting at various times after culture transfer to $37^{\circ} \mathrm{C}$. As reported previously, Maf1-7A is constitutively dephosphorylated whereas wild-type Maf1 remains phosphorylated upon this transition (Supplemental Fig. S3B). Moreover, total tRNA is reduced in Maf1-7A mutant to the same extend as previously identified using other experimental conditions (Supplemental Fig. S3C; Huber et al. 2009).

Having established the expected properties of Maf1-7A we studied its effect on the growth of trm $4 \Delta$ trm $8 \Delta$ mutant at elevated temperature. Notably, expression of Maf1-7A in both trm4 4 trm $8 \Delta$ maf $1 \Delta$ and trm $4 \Delta$ trm $8 \Delta$ cells resulted in suppression of their temperature-sensitive phenotype (Fig. 4A). Additionally plasmid encoding Maf1-7A suppressed the temperaturesensitive phenotype of tan $1 \Delta$ trm $44 \Delta$ mutant related to increased turnover of tRNA ${ }^{\text {Ser(CGA) }}$ and tRNA ${ }^{\text {Ser(UGA) }}$ (Supplemental Fig. S4).

To further characterize effect of Maf1-7A, we investigated whether suppression was linked to stabilization of hypomodified tRNA. By Northern blotting we compared levels of tRNA $\mathrm{Val}(\mathrm{AAC})$

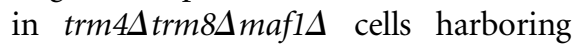
control vector and centromeric plasmids encoding wild-type Maf1 or Maf1-7A (Fig. 4B). Before the temperature shift level of $\mathrm{tRNA}^{\mathrm{Val}(\mathrm{AAC})}$ is slightly higher in control cells lacking Maf1. Following 2-h incubation of cells at $37^{\circ} \mathrm{C}, \sim 60 \%$ of tRNA ${ }^{\text {Val(AAC) }}$ was, however, degraded and the extent of degradation was similar in the presence of wild-type Mafl expressed at basal level. Indeed tRNA ${ }^{\mathrm{Val}(\mathrm{AAC})}$ was significantly more stable in the presence of Maf1-7A since only $40 \%$ was degraded. 
A
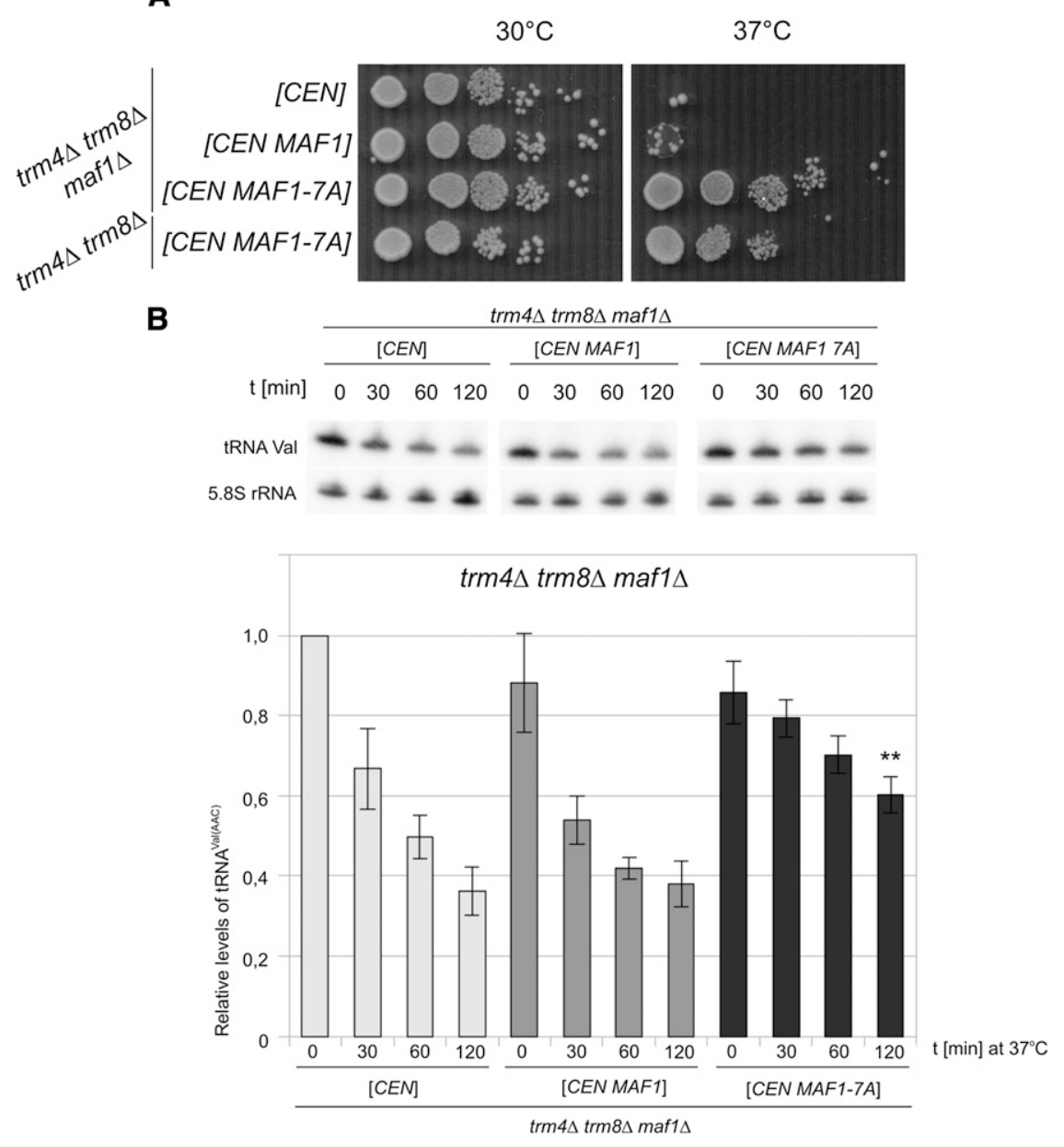

FIGURE 4. Maf1-7A mutant suppresses thermosensitive phenotype and stabilizes

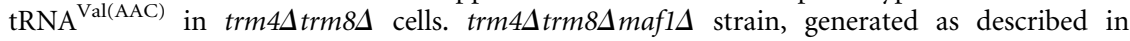
Materials and Methods, was transformed with empty plasmid pRS415 [CEN], pAH095 encoding wild-type Maf1 [CEN MAF1], or pAH247 encoding Maf1-7A mutant [CEN MAF1-7A]. (A) Transformants were grown overnight at $30^{\circ} \mathrm{C}$ in SD-ura liquid media, cultures were diluted to $\mathrm{OD}_{600}=1.0$ and serially 10 -fold diluted, spotted on YPD plate, and incubated at $30^{\circ} \mathrm{C}$ or $37^{\circ} \mathrm{C}$ for $2 \mathrm{~d}$. (B) Cells were grown in SC-Leu at $23^{\circ} \mathrm{C}$, transferred to $37^{\circ} \mathrm{C}$, and harvested as indicated. RNA was isolated and analyzed by Northern hybridization with tRNA ${ }^{\text {Val(AAC) }}$ probe and 5.8S rRNA probe (loading control). For quantification of tRNA $^{\mathrm{Val}(\mathrm{AAC})}$ levels were normalized to $5.8 \mathrm{~S}$ rRNA. Bars represent magnitude of $\mathrm{tRNA}^{\mathrm{Val}(\mathrm{AAC})}$ change calculated relative to expression in trm $4 \Delta$ trm $8 \Delta$ cells bearing empty plasmid at 0 time point. SD was calculated on the basis of three independent experiments. $P$-value $<0.005$ for the indicated probe $\left(^{* *}\right)$ was calculated relative to control strain transformed with empty plasmid $[C E N]$ at 120 time point.

These results show that the variant of Maf1 repressor, previously described in the literature as dephosphorylated and interacting with Pol III even under unstressed conditions (Huber et al. 2009), efficiently promotes stabilization of hypomodified tRNA ${ }^{\mathrm{Val}(\mathrm{AAC})}$.

\section{Decreased Pol III transcription stabilizes hypomodified tRNA ${ }^{\text {Val(AAC) }}$}

Next we asked whether repression of Pol III transcription by non-Maf1-mediated mechanisms would also stabilize
tRNA ${ }^{\mathrm{Val}(\mathrm{AAC})}$ in trm $4 \Delta$ trm $8 \Delta$ cells. First we employed rpc128-1007 mutation in the Rpc128 Pol III subunit, which halves the overall tRNA transcription (Ciesla et al. 2007). The cold-sensitive (cs) rpc128-1007 mutant was crossed with the trm4trm8s strain and the meiotic progeny was analyzed (see Materials and Methods). The observed phenotypic segregation indicated that rpc128-1007 suppressed the ts growth defect of trm $4 \Delta$ trm8 $8 \Delta$ (Fig. 5A). In accordance with the suppressed growth defect, Northern blotting revealed that the hypomodified tRNA $^{\mathrm{Val}(\mathrm{AAC})}$ was more stable in the presence of $r p c 128$ 1007 mutation, although de novo transcription is decreased, resulting in reduced level of total tRNA (Fig. 5B; Supplemental Fig. S5A). Quantification of the blots employing an intron probe showed that the rpc128-1007 mutation decreased de novo synthesis of pretRNA $^{\text {Leu }}$ in the trm $4 \Delta$ trm $8 \Delta$ strain by $40 \%$. The steady state level of tRNA $^{\mathrm{Val}(\mathrm{AAC})}$ at the permissive temperature was also significantly lower in

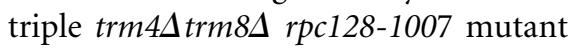
( $\sim 75 \%$ of that in double trm $4 \Delta$ trm $8 \Delta$ mutant). However, following the temperature shift, tRNA ${ }^{\text {Val(AAC) }}$ level was reduced only by $20 \%$ in trm $4 \Delta$ trm $8 \Delta$ rpc128-1007 in contrast to the reduction exceeding $70 \%$ in trm $4 \Delta$ trm $8 \Delta$.

As a yet another approach we decreased Pol III transcription by partial inactivation of its Rpc17 subunit through expression of $\mathrm{RPC} 17$ gene cloned under the regulated $t_{e t} \mathrm{O}_{7}$ promoter. Addition of doxycycline to the growth medium decreased the level of Rpc17 protein and reduced tRNA transcription whereas in the absence of the antibiotic Pol III was unaffected (Ferri et al. 2000). To monitor the effect of Rpc17 level on the stability of hypomodified tRNA $^{\mathrm{Val}(\mathrm{AAC})}$ we created a trm $4 \Delta \operatorname{trm} 8 \Delta$ strain expressing $R P C 17$ from the $t e t \mathrm{O}_{7}$ promoter. In the presence of doxycycline, Northern blot with an intron probe of pre-tRNA ${ }^{\text {Leu }}$ indicated that repression of Rpc17 expression impaired Pol III transcription and reduced tRNA levels (Fig. 5D; Supplemental Fig. S5B). Northern blotting revealed also that tRNA $^{\text {Val(AAC) }}$ was mostly degraded when the Rpc17 level was high in the absence of doxycycline but was more than twice stable when Rpc17 expression and Pol III activity were decreased in the presence of doxycycline. In keeping with the 
tRNA ${ }^{\text {Val(AAC) }}$ stabilization the $t s$ phenotype of the

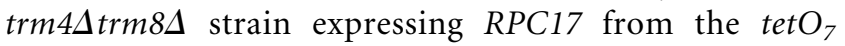
promoter was suppressed in the presence of doxycycline (Fig. 5C).

Altogether these results clearly indicate an inverse relationship between the stability of hypomodified tRNA ${ }^{\text {Val(AAC) }}$ and the activity of Pol III-directed transcription.

\section{DISCUSSION}

Our interest in the global regulation of tRNA levels, involving transcription, maturation and decay, led us to screen for gene products that control the stability of hypomodified tRNA ${ }^{\operatorname{Val}(A A C)}$. We anticipated that we would uncover genes compensating for the lowered steady state level and specific modifications missing in $\mathrm{tRNA}^{\mathrm{Val}(\mathrm{AAC})}$, in addition to those involved in indirect mechanisms affecting tRNA decay. As anticipated, most of the insert cloned contained one of the thirteen copies of genes encoding tRNA $^{\mathrm{Val}(\mathrm{AAC})}$. Surprisingly, no genes encoding tRNAmethyltransferases were uncovered. Actually, two other categories of gene products were identified: those affecting global tRNA transcription-Maf1 and truncated Pol III subunit Rpc160-and those directly interacting with tRNA $^{\mathrm{Val}(\mathrm{AAC})}$-elongation factor eEF1A and valyl-tRNA synthetase.

In yeast, Maf1 is the only known general and global negative regulator of Pol III which mediates several signaling pathways (Upadhya et al. 2002). Mafl inhibits tRNA transcription via a mechanism that depends on the dephosphorylation and nuclear accumulation of Maf1 followed by its physical association with Pol III at tRNA genes. Conversely, Maf1 phosphorylation occurs in favorable growth conditions and is linked with its cytoplasmic localization (Moir et al. 2006; Oficjalska-Pham et al. 2006; Roberts et al. 2006).

We have shown recently that Mafl indirectly affects maturation of tRNA precursors (Karkusiewicz et al. 2011). End-matured intron-containing pre-tRNAs accumulate in cells lacking Mafl due to saturation of processing machinery by the increased amounts of primary transcripts. Here we have shown that the phenotype of the trm $4 \Delta$ trm $8 \Delta$ mutant can be suppressed by overexpression of the Maf1encoding gene or, more efficiently, expression of the dephosphorylated Maf1-7A mutant that constitutively binds the Pol III complex and reduce tRNA transcription (Huber et al. 2009). The suppression by overexpressed Maf1encoding gene is accompanied by a twofold stabilization of hypomodified tRNA ${ }^{\operatorname{Val}(\mathrm{AAC})}$, while Maf1-7A mutant with reduced basal tRNA levels due to an increased capacity of Maf1-7A to bind and inhibit Pol III gives even stronger effect. Selection of a plasmid containing a 5 '-terminal part of the RPC160 gene as an autonomous suppressor of the trm $4 \Delta$ trm8 8 defect was anticipated and validates the Maf1mediated suppression. Interaction of Maf1 with the Rpc160 subunit of Pol III has been documented both genetically and by structural analysis of Pol III with Maf1 (Pluta et al. 2001; Vannini et al. 2010). Overexpression of a 5 '-terminal fragment as well as point mutations in the RPC160 gene were identified previously as suppressors of the maf1 $\Delta$ growth phenotype. That suppression was accompanied by reduction of tRNA levels in maf1 $\Delta$ cells (Boguta et al. 1997; Pluta et al. 2001). In view of those earlier data, the similar suppressor actions of Mafl and the N-terminal part of Rpc160 on the trm $4 \Delta$ trm $8 \Delta$ growth defect were expected.

The increased stability of hypomodified tRNA ${ }^{\mathrm{Val}(\mathrm{AAC})}$ caused by Mafl activation raised the idea that the observed stabilization could be related to Maf1-mediated repression of Pol III transcription. Initially this hint seemed to be inconsistent with the previous observation that cells treated with the thiolutin, Pol III inhibitor, carry out the tRNA degradation at the same rate (Chernyakov et al. 2008b). However thiolutin inhibits all RNA polymerases (Tipper 1973), exerting side effects which in turn possibly influence tRNA stabilization. Moreover, cells treated with thiolutin for a short time have stopped pre-tRNA transcription, but levels of mature tRNAs are not affected (Chernyakov et al. $2008 \mathrm{~b})$. In favor of coupling tRNA transcription to tRNA decay we found that tRNA ${ }^{\operatorname{Val}(\mathrm{AAC})}$ in trm4 4 trm $8 \Delta$ cells was stabilized upon Pol III inhibition, either in a Mafl-dependent manner or when directly down-regulating general tRNA transcription by two means: by introduction of the rpc1281007 mutation in the Pol III subunit Rpc128 or by decreasing expression of another subunit, Rpc17. Regardless of the approach used, the reduced Pol III activity and, in consequence, lower levels of total tRNA brought about a significant stabilization of the hypomodified tRNA ${ }^{\text {Val(AAC) }}$.

Proposed mechanism that could account for the effect of Pol III inhibition on stabilization of $\mathrm{tRNA}^{\mathrm{Val}(\mathrm{AAC})}$ is that, in the context of a globally lower mature tRNA level, tRNA $^{\mathrm{Val}(\mathrm{AAC})}$ could more efficiently compete for tRNAinteracting proteins helping it achieve proper conformation and/or protecting it from nucleases. We favor the latter interpretation because at least two of the multicopy suppressors of the trm $4 \Delta$ trm $8 \Delta$ mutant selected in our screen encoded tRNA-interacting proteins, eEF1A and valyltRNA synthetase, and overexpression of either of those genes also stabilized tRNA ${ }^{\mathrm{Val}(\mathrm{AAC})}$.

Taken together, our studies propose two alternative ways by which RTD degradation could be limited: a decrease in tRNA synthesis and an increase in the levels of specific tRNA-interacting proteins protecting tRNA from degradation. According to our model (Fig. 6), mature tRNAs compete with each other for interacting proteins and thus the availability of some such proteins becomes limiting. Due to the missing $\mathrm{m}^{7} \mathrm{G}_{46}$ and $\mathrm{m}^{5} \mathrm{C}_{49}$ modifications, the tertiary structure of the acceptor and $\mathrm{T}$ stems in tRNA ${ }^{\mathrm{Val}(\mathrm{AAC})}$ is imperfect or less stable (reference in Whipple et al. 2011) and, therefore, the interaction of this tRNA with proteins is compromised. It would be a poorer competitor for valyl- 
A

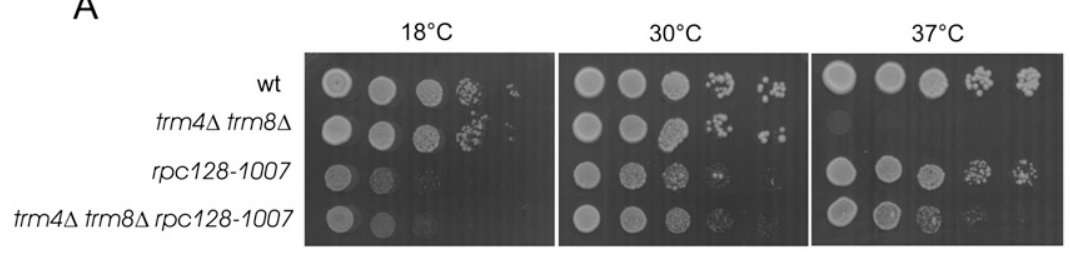

B
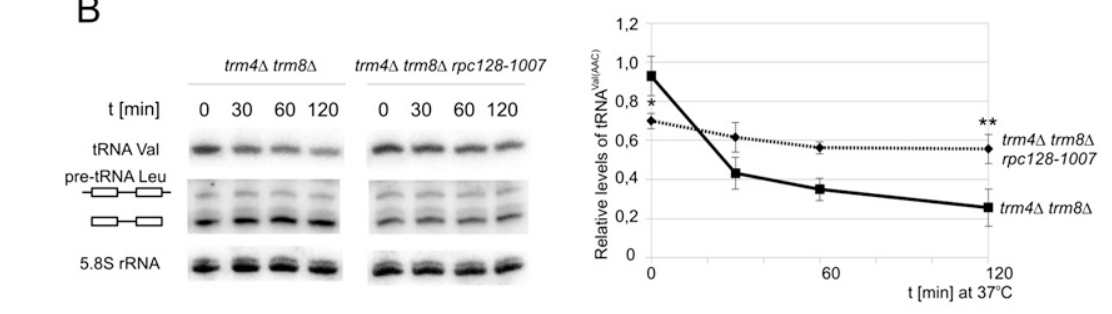

C
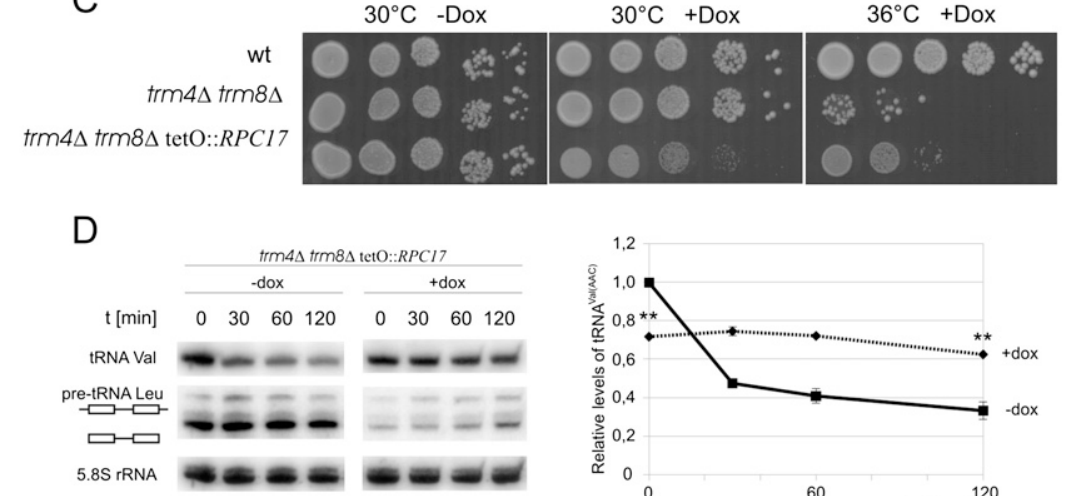

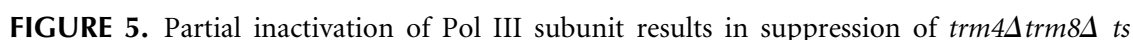
phenotype and tRNA ${ }^{\text {Val(AAC) }}$ stabilization. (A) Cold-sensitive rpc128-1007 mutation in Pol III subunit Rpc128 was introduced to trm $4 \Delta$ trm $8 \Delta$ strain by genetic cross, resulting in triple

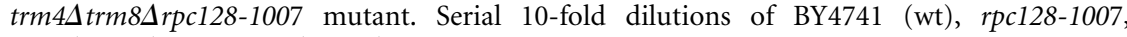

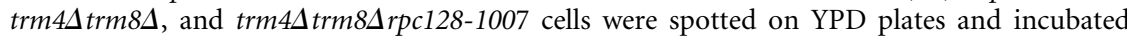
at indicated temperatures. $(B)$ Cells were grown in $\mathrm{YPD}$ to $\mathrm{OD}_{600}=1.0$ at $30^{\circ} \mathrm{C}$, transferred to $37^{\circ} \mathrm{C}$, and harvested as indicated. RNA was isolated and examined by Northern hybridization with probe specific for tRNA ${ }^{\text {Val (AAC) }}$. Plots represent levels of tRNA ${ }^{\text {al(AAC) }}$ normalized to loading control (5.8S rRNA). The magnitude of change was calculated relative to expression in trm $4 \Delta$ trm $8 \Delta$ strain at 0 time point. SD was calculated on the basis of three independent experiments. $P$-values $-P<0.01$ for the probe indicated with a single asterisk $\left.{ }^{*}\right)$ and $P<$ 0.005 for the probe indicated with two asterisks $\left({ }^{*}\right)$-were calculated relative to control strain at each time point. $(C)$ Gene encoding Rpc17 subunit of Pol III was cloned in trm4 4 trm8s strain under the regulated $t e t O_{7}$ promoter to allow down-regulation of $R P C 17$ expression by

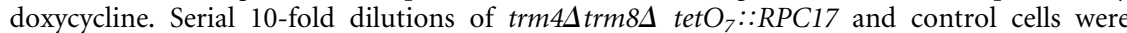
spotted to YPD plate containing $5 \mu \mathrm{g} / \mathrm{mL}$ of doxycycline and incubated for $2 \mathrm{~d}$ at indicated temperatures. Phenotypic suppression of the ts phenotype trm $4 \Delta$ trm $8 \Delta$ was most efficient at

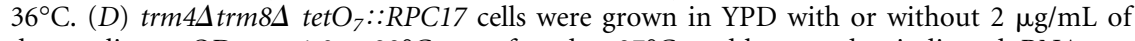
doxycycline to $\mathrm{OD}_{600}=1.0$ at $23^{\circ} \mathrm{C}$, transferred to $37^{\circ} \mathrm{C}$, and harvested as indicated. RNA was isolated and examined by Northern hybridization with probe specific for tRNA ${ }^{\mathrm{Val}(\mathrm{AAC})}$. Plots represent levels of RNA ${ }^{\text {Val(AAC) }}$ normalized to loading control (5.8S rRNA). The magnitude of change was calculated relative to expression in culture without doxycycline at zero $(0)$ time point. SD was calculated on the basis of three independent experiments. $P$-values $-P<0.005$ for the probes indicated with two asterisks $\left(^{* *}\right)$-were calculated relative to control strain at each time point.

match(es). Overexpression of limiting protein partner(s) would reduce the same effect and we suggest that this explains the suppression by valyl-tRNA synthetase and eEF1A. On the other hand, the altered structure of hypomodified tRNA ${ }^{\mathrm{Val}(\mathrm{AAC})}$ makes it more susceptible to degradation by $5^{\prime} \rightarrow 3^{\prime}$ exonucleases. This instability may also lead to isomerization of the acceptor stem, which is then subject to double CCA addition and can also be polyadenylated at the $3^{\prime}$ end like observed for hypomodified tRNA ${ }^{\text {Ser(CGA) }}$ (Wilusz et al. 2011).

How does eEF1A prevent degradation of the unstable, hypomodified tRNA ${ }^{\mathrm{Val}(\mathrm{AAC})}$ by RTD degradation? eEF1A is a homolog of E. coli EF-Tu, which interacts with the acceptor stem of aminoacyl-tRNA (Stark et al. 2002; Valle et al. 2002). Here we assume that eEF1A and $5^{\prime} \rightarrow 3^{\prime}$ exonucleases interact with the partially unstable tRNA ${ }^{\mathrm{Val}(\mathrm{AAC})}$ in a competitive manner. Fine-tuning of the competitive interaction between decay enzymes and other tRNA-binding proteins could be significant in tRNA evolution, both at the level of nucleotide sequence and nucleotide modifications. The binding of EF-Tu with aatRNA depends on the sequence and structure of $\mathrm{T}$ stem (Schrader et al. 2011). An aa-tRNA has to bind to EF$\mathrm{Tu}$ with sufficient strength to form a complex with the ribosome, but weakly enough to allow the aa-tRNA to be released during decoding. Introduction of a T-stem sequence from tightly binding tRNA to a weakly binding one results in very low rate of peptide bond formation. Assuming that improper binding of tRNA with eEF1A increases the accessibility of this tRNA to $5^{\prime} \rightarrow 3^{\prime}$ exonucleases, the RTD-mediated degradation could provide a positive tRNA selection in evolution.

\section{MATERIALS AND METHODS}

\section{Strains, plasmids, and media}

tRNA synthetase or eEF1A than other tRNAs. A globally decreased tRNA synthesis would relief that competition by decreasing the overall tRNA:protein ratio, thereby allowing even the imperfect, hypomodified tRNA ${ }^{\mathrm{Val}(\mathrm{AAC})}$ to find its

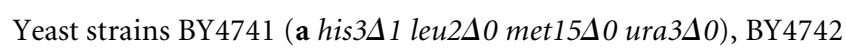
( $\boldsymbol{\alpha}$ his $3 \Delta 1$ leu2 $\Delta 0$ lys $2 \Delta 0$ ura3 $\Delta 0$ ), and isogenic trm $4 \Delta$ and trm $8 \Delta$ were purchased from Euroscarf. The trm $4 \Delta$ trm $8 \Delta$ haploid and homozygous diploid mutants, $\Delta \mathrm{TT} 1 \mathrm{~A}$ ( $\alpha$ his $3 \Delta 1$ leu $2 \Delta 0$ lys $2 \Delta 0$ 


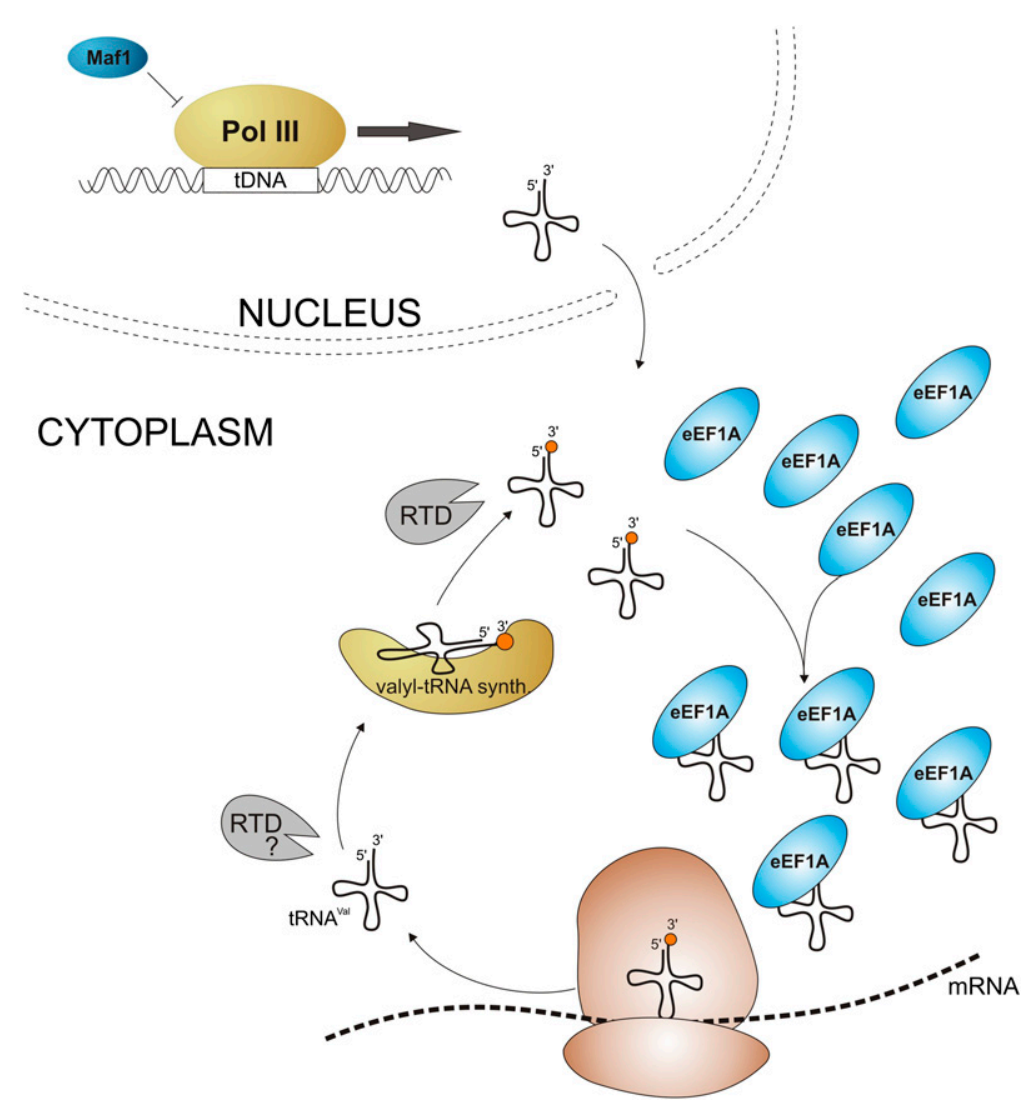

FIGURE 6. Model for eEF1A-mediated protection of tRNA ${ }^{\mathrm{Val}(\mathrm{AAC})}$ from RTD degradation. Mature, hypomodified tRNA ${ }^{\mathrm{Val}(\mathrm{AAC})}$ pool is aminoacylated by valyl-tRNA synthetase, bound by eEF1A, and delivered to ribosomes. A shift to restrictive conditions results in degradation of hypomodified tRNA ${ }^{\mathrm{Val}(\mathrm{AAC})}$ by RTD pathway. It is unknown whether hypomodified tRNA can be efficiently charged and if it could be degraded prior to aminoacylation. Nevertheless, elevated population of eEF1A molecules favors their binding to tRNA ${ }^{\text {Val(AAC) }}$ and prevents tRNA $^{\text {Val(AAC) }}$ interacting with nucleases. Increased availability of tRNA-free eEF1A molecules may be achieved by increased expression of TEF1 gene or decreased global tRNA synthesis by Pol III inhibition.

met $15 \Delta 0$ ura3s0 trm $4 \Delta:: k a n M X 4$ trm $8 \Delta:: k a n M X 4)$ and $\Delta$ TT $2 \mathrm{n}$

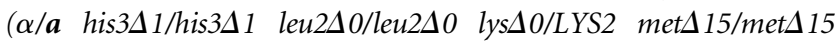
ura3 $\Delta 0 /$ ura3 $\Delta 0$ trm $4 \Delta:: k a n M X 4 /$ trm $4 \Delta:: k a n M X 4$ trm $8 \Delta::$ kanMX4/trm8s::kanMX4), respectively, were obtained by genetic crosses. Single copy of endogenous MAF1 gene in $\triangle \mathrm{TT} 2 \mathrm{n}$ was disrupted by $U R A 3$ cassette and the resulted diploid (relevant

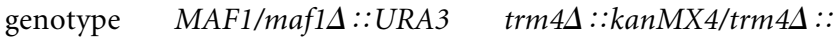

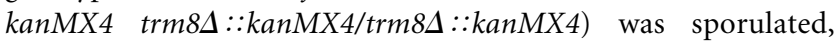
resulting in 2:2 segregation of viable MAF1 Ura- spores. Ura+ spores containing maf1::URA3 allele germinated only at $18^{\circ} \mathrm{C}$ and showed a 12 -h lag in growth at $30^{\circ} \mathrm{C}$. This growth defect was complemented with $M A F 1$ gene supplied from plasmid. To generate

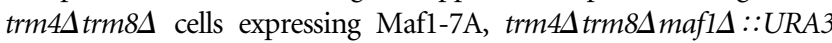
spore clone was transformed with single copy plasmid pAH247 (pRS415::MAF1-7A [S90A, S101A, S177A, s178A, S179A, S209A, S210A]; Huber et al. 2009).

BY derivatives trm $44 \Delta$ and $\tan 1 \Delta$ were purchased from Euroscarf. tan $1 \Delta$ trm $44 \Delta$ mutant was generated by genetic cross.

Triple rpc128-1007 trm4 4 trm8s mutant was isolated from among

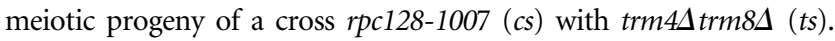
Thirteen tetrads were analyzed for kanamycin resistance and growth at $37^{\circ} \mathrm{C}$ and $18^{\circ} \mathrm{C}$. The cs phenotype indicating rpc128-1007 segregated 2:2. Double trm $4 \Delta$ trm $8 \Delta$ deletions were identified among kanamycin resistance spore clones by PCR analysis of genomic DNA. All spore clones

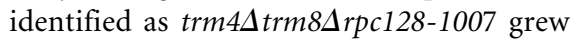
at $37^{\circ} \mathrm{C}$.

The pCM189[RPC17] plasmid encoding repressible Rpc17 Pol III subunit was created in E. coli strain $\mathrm{MH} 1$ from pCMc17 (Ferri et al. 2000) using pCM189 (Euroscarf) as backbone. Single copy of endogenous

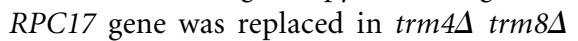
homozygous diploid yeast strain by HIS3 cassette and the obtained strain (relevant genotype RPC17/rpc17A::HIS3 trm $4 \Delta::$

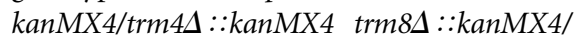
trm8s::kanMX4) was transformed with pCM189[RPC17] plasmid and sporulated. The growth of spore clones containing rpc174::HIS3 allele and pCM189[RPC17] plasmid was sensitive to doxycycline $(2 \mu \mathrm{g} /$ $\mathrm{mL}$ in solid YPD) but resistant to elevated temperature.

Yeast YPD or YPGly complete media contained $1 \%$ yeast extract, $2 \%$ peptone, and $2 \%$ glucose or $2 \%$ glycerol, respectively. The minimal media, SC-ura or SC-leu, containing $0.67 \%$ yeast nitrogen base and $2 \%$ glucose or glycerol were supplemented with all necessary requirements without uracil or leucine, respectively. Solid media contained 2\% agar. All reagents were from Difco.

\section{Cloning of trm4strm8s defect suppressors}

Suppressors of trm $4 \Delta$ trm $8 \Delta$ were cloned by complementation of the ts growth phenotype on YPD medium. trm $4 \Delta$ trm $8 \Delta$ strain was transformed with yeast genebank on multicopy pFL44L vector provided by F. Lacroute (Stettler et al. 1993) and plated on SC-ura. About 5000 URA+ transformants were replicated onto YPD medium and incubated at $37^{\circ} \mathrm{C}$ for $7 \mathrm{~d}$, with growth monitored every day. POP5 was subcloned from $\mathrm{p} 47$ plasmid containing genomic fragment of chromosome I (coordinates $80144-84902$ ) by ligation of NarI-NarI fragment to pFL44L empty vector.

\section{Northern analysis}

Cells were grown according to Alexandrov et al. (2006) with modification. One-hundred milliliters of culture grown at $23^{\circ} \mathrm{C}$ to $\mathrm{OD}_{600}=1.0$ was rapidly mixed with $400 \mathrm{~mL}$ of media preheated to $42^{\circ} \mathrm{C}$. The cultures were further incubated at $37^{\circ} \mathrm{C}$ and harvested at time points indicated by centrifugation in a rotor preheated to $37^{\circ} \mathrm{C}$. For cold-sensitive strains with rpc128-1007 mutation or overexpressed MAF1 $100 \mathrm{~mL}$ of culture grown at $30^{\circ} \mathrm{C}$ to $\mathrm{OD}_{600}=$ 1.0 was rapidly mixed with $400 \mathrm{~mL}$ of media preheated to $40^{\circ} \mathrm{C}$. Repression of RPC17 gene in liquid cultures was done by adding 
doxycycline (Sigma) to $2 \mu \mathrm{g} / \mathrm{mL}$ final concentration and further incubation for $\sim 24 \mathrm{~h}$ to $\mathrm{OD}_{600}=1.0$. RNA was extracted and hybridized as described previously (Graczyk et al. 2011). The following probes were employed: tRNA ${ }^{\mathrm{Val}(\mathrm{AAC})}$ : TGGTGATTTCGCC CAGGA; tRNA ${ }^{\mathrm{Leu}(\mathrm{CAA})}$ intron: TATTCCCACAGTTTGCGGTCA; 5.8S rRNA: GCGTTGTTCATCGATGC. After hybridization blots were washed $3 \times 10 \mathrm{~min}$ with $1 \times$ SSC, $1 \%$ SDS and $2 \times 10 \mathrm{~min}$ with $0.5 \times \mathrm{SSC}, 0.1 \%$ SDS at $37^{\circ} \mathrm{C}$, exposed to a phosphoimager screen (Fujifilm). RNA was quantified using FLA-7000 PhosphoImager (Fujifilm). Band intensities were quantified using Multi Gauge V3.0 Software. The statistical significance was computed using a $t$-test as implemented in the OpenOffice.org environment.

\section{Western analysis}

Cells were broken and proteins isolated as described previously (Towpik et al. 2008). Protein extracts were separated on $10 \%$ (acrylamide:bisacrylamide 33.5:0.3) SDS-PAGE and hybridized with Maf1-specific antibody as described previously (Gajda et al. 2010).

\section{SUPPLEMENTAL MATERIAL}

Supplemental material is available for this article.

\section{ACKNOWLEDGMENTS}

We thank Olivier Lefebvre and Damian Graczyk for correction of the manuscript and Alexandre Huber for kindly providing Maf1-7A encoding plasmid. This work was supported by National Center of Science UMO-2011/01/N/NZ1/03461 and by the European Union in the framework of European Social Fund through the Warsaw University of Technology Development Programme.

Received April 4, 2012; accepted July 3, 2012.

\section{REFERENCES}

Alexandrov A, Chernyakov I, Gu W, Hiley SL, Hughes TR, Grayhack EJ, Phizicky EM. 2006. Rapid tRNA decay can result from lack of nonessential modifications. Mol Cell 21: 87-96.

Anderson JS, Parker RP. 1998. The $3^{\prime}$ to $5^{\prime}$ degradation of yeast mRNAs is a general mechanism for mRNA turnover that requires the SKI2 DEVH box protein and $3^{\prime}$ to $5^{\prime}$ exonucleases of the exosome complex. EMBO J 17: 1497-1506.

Boguta M, Graczyk D. 2011. RNA polymerase III under control: Repression and de-repression. Trends Biochem Sci 36: 451-456.

Boguta M, Czerska K, Zoladek T. 1997. Mutation in a new gene MAF1 affects tRNA suppressor efficiency in Saccharomyces cerevisiae. Gene 185: 291-296.

Chernyakov I, Baker MA, Grayhack EJ, Phizicky EM. 2008a. Chapter 11. Identification and analysis of tRNAs that are degraded in Saccharomyces cerevisiae due to lack of modifications. Methods Enzymol 449: 221-237.

Chernyakov I, Whipple JM, Kotelawala L, Grayhack EJ, Phizicky EM. 2008b. Degradation of several hypomodified mature tRNA species in Saccharomyces cerevisiae is mediated by Met22 and the $5^{\prime}-3^{\prime}$ exonucleases Rat1 and Xrn1. Genes Dev 22: 1369-1380.

Ciesla M, Towpik J, Graczyk D, Oficjalska-Pham D, Harismendy O, Suleau A, Balicki K, Conesa C, Lefebvre O, Boguta M. 2007. Maf1 is involved in coupling carbon metabolism to RNA polymerase III transcription. Mol Cell Biol 27: 7693-7702.

Desai N, Lee J, Upadhya R, Chu Y, Moir RD, Willis IM. 2005. Two steps in Maf1-dependent repression of transcription by RNA polymerase III. J Biol Chem 280: 6455-6462.

Ferri ML, Peyroche G, Siaut M, Lefebvre O, Carles C, Conesa C, Sentenac A. 2000. A novel subunit of yeast RNA polymerase III interacts with the TFIIB-related domain of TFIIIB70. Mol Cell Biol 20: $488-495$.

Gajda A, Towpik J, Steuerwald U, Muller CW, Lefebvre O, Boguta M. 2010. Full repression of RNA polymerase III transcription requires interaction between two domains of its negative regulator Maf1. J Biol Chem 285: 35719-35727.

Graczyk D, Debski J, Muszynska G, Bretner M, Lefebvre O, Boguta M. 2011. Casein kinase II-mediated phosphorylation of general repressor Maf1 triggers RNA polymerase III activation. Proc Natl Acad Sci 108: 4926-4931.

Huber A, Bodenmiller B, Uotila A, Stahl M, Wanka S, Gerrits B, Aebersold R, Loewith R. 2009. Characterization of the rapamycinsensitive phosphoproteome reveals that Sch9 is a central coordinator of protein synthesis. Genes Dev 23: 1929-1943.

Jha S, Dutta A. 2009. RVB1/RVB2: Running rings around molecular biology. Mol Cell 34: 521-533.

Kadaba S, Krueger A, Trice T, Krecic AM, Hinnebusch AG, Anderson J. 2004. Nuclear surveillance and degradation of hypomodified initiator tRNAMet in S. cerevisiae. Genes Dev 18: 1227-1240.

Karkusiewicz I, Turowski TW, Graczyk D, Towpik J, Dhungel N, Hopper AK, Boguta M. 2011. Maf1 protein, repressor of RNA polymerase III, indirectly affects tRNA processing. $J$ Biol Chem 286: 39478-39488.

Kotelawala L, Grayhack EJ, Phizicky EM. 2008. Identification of yeast tRNA $\mathrm{Um}_{44}$ 2'-O-methyltransferase (Trm44) and demonstration of a Trm44 role in sustaining levels of specific tRNA ${ }^{\text {Ser }}$ species. RNA 14: 158-169.

Kuai L, Fang F, Butler JS, Sherman F. 2004. Polyadenylation of rRNA in Saccharomyces cerevisiae. Proc Natl Acad Sci 101: 8581-8586.

Marshall L, Kenneth NS, White RJ. 2008. Elevated tRNA ${ }_{i}^{\text {Met }}$ synthesis can drive cell proliferation and oncogenic transformation. Cell 133: 78-89.

Mateyak MK, Kinzy TG. 2010. eEF1A: Thinking outside the ribosome. J Biol Chem 285: 21209-21213.

Moir RD, Lee J, Haeusler RA, Desai N, Engelke DR, Willis IM. 2006. Protein kinase A regulates RNA polymerase III transcription through the nuclear localization of Maf1. Proc Natl Acad Sci 103: 15044-15049.

Oficjalska-Pham D, Harismendy O, Smagowicz WJ, Gonzalez de Peredo A, Boguta M, Sentenac A, Lefebvre O. 2006. General repression of RNA polymerase III transcription is triggered by protein phosphatase type 2A-mediated dephosphorylation of Maf1. Mol Cell 22: 623-632.

Phizicky EM, Hopper AK. 2010. tRNA biology charges to the front. Genes Dev 24: 1832-1860.

Pluta K, Lefebvre O, Martin NC, Smagowicz WJ, Stanford DR, Ellis SR, Hopper AK, Sentenac A, Boguta M. 2001. Maflp, a negative effector of RNA polymerase III in Saccharomyces cerevisiae. Mol Cell Biol 21: 5031-5040.

Roberts DN, Wilson B, Huff JT, Stewart AJ, Cairns BR. 2006. Dephosphorylation and genome-wide association of Maf1 with Pol III-transcribed genes during repression. Mol Cell 22: 633644.

Schrader JM, Chapman SJ, Uhlenbeck OC. 2011. Tuning the affinity of aminoacyl-tRNA to elongation factor Tu for optimal decoding. Proc Natl Acad Sci 108: 5215-5220.

Stark H, Rodnina MV, Wieden HJ, Zemlin F, Wintermeyer W, van Heel M. 2002. Ribosome interactions of aminoacyl-tRNA and elongation factor Tu in the codon-recognition complex. Nat Struct Biol 9: 849-854.

Stettler S, Chiannilkulchai N, Hermann-Le Denmat S, Lalo D, Lacroute F, Sentenac A, Thuriaux P. 1993. A general suppressor of RNA 


\section{Turowski et al.}

polymerase I, II and III mutations in Saccharomyces cerevisiae. Mol Gen Genet 239: 169-176.

Tipper DJ. 1973. Inhibition of yeast ribonucleic acid polymerases by thiolutin. J Bacteriol 116: 245-256.

Towpik J, Graczyk D, Gajda A, Lefebvre O, Boguta M. 2008. Derepression of RNA polymerase III transcription by phosphorylation and nuclear export of its negative regulator, Mafl. J Biol Chem 283: 17168-17174.

Upadhya R, Lee J, Willis IM. 2002. Maf1 is an essential mediator of diverse signals that repress RNA polymerase III transcription. Mol Cell 10: 1489-1494.

Valle M, Sengupta J, Swami NK, Grassucci RA, Burkhardt N, Nierhaus KH, Agrawal RK, Frank J. 2002. Cryo-EM reveals an active role for aminoacyl-tRNA in the accommodation process. EMBO J 21: 3557-3567.
Vannini A, Ringel R, Kusser AG, Berninghausen O, Kassavetis GA, Cramer P. 2010. Molecular basis of RNA polymerase III transcription repression by Maf1. Cell 143: 59-70.

Whipple JM, Lane EA, Chernyakov I, D’Silva S, Phizicky EM. 2011. The yeast rapid tRNA decay pathway primarily monitors the structural integrity of the acceptor and T-stems of mature tRNA. Genes Dev 25: 1173-1184.

White RJ. 2004. RNA polymerase III transcription and cancer. Oncogene 23: 3208-3216.

Wilusz JE, Whipple JM, Phizicky EM, Sharp PA. 2011. tRNAs marked with CCACCA are targeted for degradation. Science 334: 817-821.

Wyers F, Rougemaille M, Badis G, Rousselle JC, Dufour ME, Boulay J, Regnault B, Devaux F, Namane A, Seraphin B, et al. 2005. Cryptic pol II transcripts are degraded by a nuclear quality control pathway involving a new poly(A) polymerase. Cell 121: 725-737. 

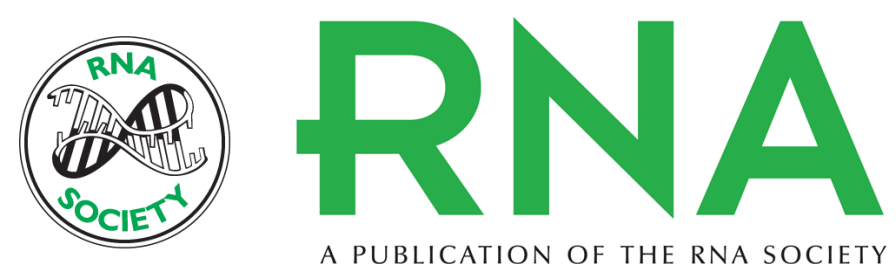

A PUBLICATION OF THE RNA SOCIETY

\section{Maf1-mediated repression of RNA polymerase III transcription inhibits tRNA degradation via RTD pathway}

Tomasz W. Turowski, Iwona Karkusiewicz, Justyna Kowal, et al.

RNA 2012 18: 1823-1832 originally published online August 23, 2012

Access the most recent version at doi:10.1261/rna.033597.112

Supplemental http://rnajournal.cshlp.org/content/suppl/2012/08/03/rna.033597.112.DC1
Material

References This article cites 36 articles, 22 of which can be accessed free at:

http://rnajournal.cshlp.org/content/18/10/1823.full.html\#ref-list-1

License

Email Alerting Receive free email alerts when new articles cite this article - sign up in the box at the Service top right corner of the article or click here. 